\title{
PERFIL DAS ENFERMEIRAS QUE ATUAM NA ASSISTÊNCIA A GESTANTE, PARTURIENTE E PUÉRPERA, EM INSTITUIÇÕES DE SOROCABA/SP (1999) ${ }^{1}$
}

\author{
Carmen Lucia Cipullo Gardenal ${ }^{2}$ \\ Ivanil Parreira ${ }^{3}$ \\ Janie Maria de Almeida ${ }^{4}$ \\ Valdina Marins Pereira ${ }^{5}$
}

Gardenal CLC, Parreira I, Almeida JM, Pereira VM. Perfil das enfermeiras que atuam na assistência à gestante, parturiente e puérpera, em instituições de Sorocaba/SP (1999). Rev Latino-am Enfermagem 2002 julho-agosto; 10(4):478-84.

O estudo objetivou: alistar as instituições que assistem à gestante, parturiente e puérpera; identificar as enfermeiras que participam dessa assistência, as atividades por elas realizadas, os problemas enfrentados e o grau de satisfação no trabalho. Nas 35 instituições existentes em Sorocaba, foram entrevistadas 74 enfermeiras, sendo $27 \%$ especializadas e $28 \%$ habilitadas em Obstetrícia; as demais não realizaram nenhum curso na área. Os principais problemas relatados foram falta de segurança para realizar a consulta pré-natal, restrição de pessoal e de material e acúmulo de funções. Setenta e três por cento das entrevistadas referiram estar satisfeitas no trabalho.

DESCRITORES: enfermagem obstétrica, assistência ao paciente, enfermagem, saúde da mulher

\section{PROFILE OF NURSES WORKING IN INSTITUTIONS WHERE CARE TO PREGNANT, PARTURIENT AND PUERPERAL WOMEN WAS GIVEN IN THE CITY OF SOROCABA - SP, BRAZIL (1999)}

This study aimed at verifying the institutions where pregnant, parturient and puerperal women were given care. It also aimed at identifying the nurses who participate in such caregiving, the activities performed and the problems faced by them and their level of satisfaction at work. Seventy-four nurses were interviewed in the 35 institutions found in Sorocaba, of whom $27 \%$ were specialists and $28 \%$ were qualified obstetricians. The others had not been trained in that area. The major problems reported were the lack of skills to perform prenatal nursing consultations, shortage of staff and materials and the accumulation of. Seventythree percent of the interviewees reported to be satisfied in relation to their jobs.

DESCRIPTORS: obstetric nursing, patient care, nursing, women's health

\section{PERFIL de LAS ENFERMERAS QUE ACTUAN EN LA ATENCIÓN A LA EMBARAZADA, PARTURIENTA Y PUERPERA EN LAS INSTITUCIONES DE SOROCABASP (1999)}

El estudio tuvo como objetivo: enumerar las instituciones que atienden a las embarazadas, parturientas y puerperas; identificar las enfermeras que hacen esa atención, las tareas ejecutadas, los problemas enfrentados y el grado de satisfacción con su trabajo. En 35 instituciones fueron entrevistadas 74 enfermeras: $27 \%$ especializadas y $28 \%$ habilitadas en Obstetricia; las demás no asistieron a cursos en la especialidad. Los principales problemas encontrados fueron: la ausencia de seguridad para la realización de la consulta prenatal, la restricción de personal y de material, y la acumulación de funciones. $73 \%$ refirieron estar satisfechas con su trabajo.

DESCRIPTORES: enfermeria obstetrica, asistencia ao paciente, enfermeria, salud de la mujer

\footnotetext{
${ }^{1}$ Trabalho apresentado no $51^{\circ}$ Congresso Brasileiro de Enfermagem - Florianópolis/SC; ${ }^{2}$ Professor Assistente - Mestre. Disciplina Administração em Enfermagem; ${ }^{3}$ Professor da Disciplina Enfermagem Fundamental; ${ }^{4}$ Professor da Disciplina Enfermagem na Reprodução Humana; ${ }^{5}$ Professor Assistente Doutor da Disciplina Enfermagem na Reprodução Humana, e-mail: facaval@terra.com.br. Departamento de Enfermagem, Centro de Ciências Médicas e Biológicas da Pontifícia Universidade Católica de São Paulo
} 
O ensino da Obstetrícia, no Brasil, teve início em $1832^{(1)}$, conforme descreve a Lei de 03 de outubro desse ano, sendo, então, denominado curso de "Partos", e ministrado em Faculdades de Medicina, juntamente com outros dois cursos: o de Medicina e o de Farmácia. Já o ensino de Enfermagem surgiu 90 anos depois, com seu primeiro currículo regulamentado pelo Decreto $\mathrm{n}^{\circ} .16 .300 / 23^{(2)}$, incluindo, no seu programa, a arte de enfermeira em obstetrícia e gynecologia. Passou, até os dias de hoje, por cinco experiências curriculares, estando sempre presentes, em todas elas, os conteúdos de Enfermagem Obstétrica.

A despeito de os cursos de Enfermagem incluírem disciplinas relacionadas à assistência maternoinfantil, os cursos de Obstetrícia funcionaram paralelamente até 1972, quando a Resolução $n^{\circ} 4$ estabeleceu a fusão desses dois cursos em um único, com três fases: a pré-profissional, o tronco profissional comum e a habilitação ${ }^{(3)}$.

Em 1994, porém, a Portaria $n^{0} 1.721$, do Ministério da Educação, extinguiu as Habilitações, restando como único meio de formação específica, na área obstétrica, para Enfermeiros, a pós-graduação - lato sensu ou curso de Especialização ${ }^{(4)}$. Nessa última década, como parte da estratégia governamental para a redução da morbimortalidade perinatal, foram publicados vários dispositivos legais relacionados à área obstétrica. Embora a própria Lei do Exercício Profissional da Enfermagem, de 1986, já pontuasse, com clareza, as atribuições do enfermeiro, na assistência à mulher, no ciclo gravídico-pueperal ${ }^{(5)}$, foram as Portarias $2815 / 98^{(6)}$ e $163 / 98^{(7)}$ que vieram valorizar o trabalho do enfermeiro Obstetra e Obstetriz, provocando novas discussões sobre a função desse profissional na sociedade.

Em decorrência dessas Portarias e após quase duas décadas de desmotivação dos enfermeiros para atuarem em Obstetrícia, estamos presenciando um aumento da procura de formação específica nessa área, vista como desejável no atual cenário da assistência materno-infantil.

Tendo sido o nosso Departamento de Enfermagem escolhido para sediar o II Seminário Estadual sobre o Ensino de Enfermagem para a Assistência ao Nascimento e Parto (1999), promovido pela $A B E N F O / S P$, foi apresentado às participantes um panorama da situação de trabalho das Enfermeiras que atuam na assistência obstétrica em Sorocaba. Com essa finalidade, foram, então, delimitados os seguintes objetivos:

- Identificar as instituições prestadoras de assistência à mulher, da gestação ao puerpério, no município de Sorocaba;

- Identificar o perfil das Enfermeiras que desenvolvem funções/atividades relacionadas à Obstetrícia nessas instituições;

- Realizar um diagnóstico da prática de enfermagem relacionada ao pré-natal, pré-parto, parto e puerpério, a partir dos relatos das enfermeiras que atuam nessas áreas.

Temos em mente que esse diagnóstico deve reverter em propostas para os serviços que atendem à mulher, na cidade de Sorocaba.

\section{MÉTODOS}

A amostragem foi construída, obedecendo-se às seguintes etapas:

$1^{\mathrm{a}}$ - mapeamento das instituições de saúde da cidade de Sorocaba/SP, que prestam assistência à mulher no ciclo gravídico-puerperal: hospitalares, ambulatoriais, clínicas e Unidades Básicas de Saúde;

$2^{\mathrm{a}}$ - para cada instituição mapeada, realizou-se um levantamento do número de enfermeiras e daquelas que desenvolvem atividades no programa de saúde da mulher, especificamente, no período da gestação, parto e puerpério;

$3^{\text {a }}$ - aplicação de um formulário com 16 questões relacionadas à identificação da entrevistada, formação profissional, funções desempenhadas no momento, problemas encontrados, nível de satisfação no trabalho e perspectivas quanto ao aperfeiçoamento profissional; $4^{\mathrm{a}}$ - dessa forma, de um total de 93 enfermeiras que atuam nessas instituições, 74 enfermeiras que se encontravam na ativa no momento da aplicação do questionário, de março a junho de 1999 , foram entrevistadas. O plano de amostragem constitui-se, dessa maneira, num censo das profissionais que atuam na assistência à mulher gestante, parturiente e puérpera de Sorocaba.

O projeto da pesquisa foi aprovado pelo Comitê de Ética em Pesquisa do Centro de Ciências Médicas e Biológicas de Sorocaba, da Pontifícia Universidade 
Católica de São Paulo (CCMB-PUCSP), e foi obtida, previamente, autorização para aplicação do questionário, não apenas de cada instituição envolvida, como também das 74 enfermeiras que aceitaram responder à entrevista.

\section{RESULTADOS E DISCUSSÃO}

Pesquisamos: 6 hospitais, 26 Unidades Básicas de Saúde, 1 Unidade de Pronto Atendimento, 1 Policlínica de especialidades e o Departamento de Enfermagem do CCMB-PUCSP.

Do total de entrevistadas, observamos que, quanto à idade, $86,5 \%$, têm mais de 30 anos de idade, sendo $56,7 \%$ entre 31 e 40 anos; e $13,5 \%$, de 21 a 30 anos; por sua vez, quanto ao estado civil, $62,2 \%$ são casadas, $27,0 \%$ são solteiras. Na cidade de Sorocaba, não existe nenhum enfermeiro do sexo masculino atuando nessa área.

$\mathrm{Na}$ Tabela 1 , pode-se observar que $74 \%$ dos sujeitos da pesquisa são egressos da nossa Universidade (PUC); observamos, também, profissionais provenientes de outros estados do Brasil e até de outro país (Chile).

Tabela 1 - Distribuição numérica e percentual das enfermeiras que atuam na assistência à mulher no ciclo gravídico-puerperal, segundo a instituição em que cursaram a graduação em enfermagem, Sorocaba-SP, 1999

\begin{tabular}{lcc}
\multicolumn{1}{c}{ Instituição } & I & $\%$ \\
\hline PUC-SP & 55 & 74,3 \\
Outras escolas do Estado de São Paulo & 15 & 20,3 \\
Outras escolas do País & 3 & 4,1 \\
Outras escolas fora do País & 1 & 1,3 \\
\hline TOTAL & $\mathbf{7 4}$ & $\mathbf{1 0 0 , 0}$ \\
\hline
\end{tabular}

Ao analisarmos o tempo decorrido após a Graduação, constatamos que 33,8\% estão formadas há menos de 10 anos; destas, quinze enfermeiras $(20,3 \%)$ terminaram o curso há menos de 5 anos, período em que as habilitações já haviam sido extintas. Esse fato pode explicar a busca desse grupo pelo curso de especialização em Enfermagem Obstétrica, oferecido em 1998, pelo Centro de Ciências Médicas e Biológicas de Sorocaba, do qual participaram 11 enfermeiras graduadas há menos de cinco anos.

Mais da metade das entrevistadas (52,1\%) têm de 11 a 20 anos de profissão, e 6,8\% destas graduaram- se há mais de 30 anos; essas últimas já estão aposentadas e continuam exercendo suas funções. $O$ fato de essas profissionais estarem na ativa pode ter como uma das explicações a escassez de novos profissionais especialistas no mercado de trabalho.

Quanto à qualificação profissional das enfermeiras (Tabela 2), observamos que $54,8 \%$ das enfermeiras que trabalham na área da assistência à mulher, no ciclo gravídico-puerperal, possuem especialização ou habilitação na área. Dentre as demais, as que atuam na rede pública fizeram capacitação para desenvolverem funções da Enfermeira Obstétrica, tais como realizar o exame preventivo para o câncer cervical e a consulta pré-natal. Essa capacitação variou de 45 dias, para as admitidas há mais tempo, até dois dias, para as admitidas recentemente.

Entretanto, 33,8\% das entrevistadas possuem habilitação em outras áreas, e 10,8\% não fizeram nenhum curso após a graduação. Entre as outras especializações/ habilitações realizadas, as mais citadas pelas enfermeiras foram: Saúde Pública e Administração Hospitalar.

Tabela 2 - Distribuição numérica e percentual de enfermeiras que atuam na assistência à mulher no ciclo gravídico-puerperal, segundo as habilitações e/ou especializações, Sorocaba-SP, 1999

\begin{tabular}{lcc}
\hline \multicolumn{1}{c}{ Categoria } & II & $\%$ \\
\hline Habilitada em Obstetrícia & 19 & 25,7 \\
Especializada em Obstetrícia & 19 & 25,7 \\
HabilitadarEspecializada em outra área & 25 & 33,8 \\
Habilitada e Especializada em Obstetrícia & 3 & 4,0 \\
Nấo tern HabıEsp. em nenhuma área & 8 & 10,8 \\
\hline TOTAL & $\mathbf{7 4}$ & $\mathbf{1 0 0 , 0}$ \\
\hline
\end{tabular}

Destaca-se a predominância de egressos do curso de Habilitação/Especialização de Sorocaba (Tabela 3), deduzindo-se que o mercado de trabalho da região oferece, ainda, uma boa absorção para os profissionais de enfermagem na área obstétrica e os que aqui se graduaram estão ocupando esse mercado. Verificamos que onze das doze enfermeiras que referiram ter cursado Especialização, nos últimos cinco anos, fizeram-no na PUC-SP, em Sorocaba, em 1998. Também as cinco enfermeiras que se especializaram há mais de 30 anos, fizeram-no em Sorocaba. 
Tabela 3 - Distribuição numérica de enfermeiras habilitadas e ou especializadas na área de obstetrícia, segundo a instituição onde se habilitaram/especializaram, Sorocaba-SP, 1999

\begin{tabular}{lccc}
\hline \multicolumn{1}{c}{ Instituição } & Habiltação & Especializaçäo & Total \\
\hline PUC-SP & 18 & 17 & 35 \\
USP & 1 & 1 & 2 \\
Faculdade Adventista de & 1 & - & 1 \\
Enfermagem & & 1 & 1 \\
Universidadede Guanulhos & - & 1 & 1 \\
Universidade Sấo Camilo & - & 1 & 2 \\
Faculdade Municipal de & 1 & - & 1 \\
Araras & 1 & 1 & 1 \\
UNIFESP & - & $\mathbf{2 2}$ & $\mathbf{4 4}$ \\
Universidade do Chile & $\mathbf{2 2}$ & & \\
\hline TOTAL & &
\end{tabular}

As enfermeiras foram entrevistadas nas respectivas instituições, considerando-se essa instituição como o seu trabalho principal, pois $49,3 \%$ delas atuam em mais de um estabelecimento. A distribuição das respondentes, segundo o campo de atuação principal, está assim distribuída: $59,5 \%$ atuam na rede municipal, UBS, PA e Policlínica; $37,8 \%$, na rede Hospitalar e Maternidade; e 2,7\%, no Ensino Superior.

Das enfermeiras entrevistadas que trabalham em mais de uma instituição, 52,8\% referiram que atuam, também nesse segundo emprego, na área obstétrica, e as outras $47,2 \%$ indicaram atuar em Administração Hospitalar, Enfermagem Psiquiátrica e Programa de Saúde da Família, o que é um paradoxo, tendo em vista a necessidade do mercado de trabalho no campo da saúde materno-infantil. Por outro lado, oito dessas profissionais atuam no ensino em Obstetrícia, possibilitando a transferência de sua experiência a novas gerações de enfermeiras. Vale registrar que foi encontrada uma enfermeira habilitada em Obstetrícia, exercendo apenas funções administrativas em Unidade Básica de Saúde.

Foi também objetivo deste trabalho verificar as atividades predominantes no cotidiano das profissionais que assistem a mulher, no ciclo gravídico-puerperal (Tabela 4).

Tabela 4 - Fase do ciclo gravídico-puerperal em que as enfermeiras atuam, Sorocaba-SP, 1999

\begin{tabular}{lccccc}
\hline \multicolumn{1}{c}{ Fase em que atuam } & \multicolumn{2}{c}{ Sempre } & \multicolumn{2}{c}{ Eventualmente } \\
\hline No puerpério & 25 & $33,8 \%$ & 42 & $56,7 \%$ \\
No pré-natal & 10 & $13,5 \%$ & 36 & $48,6 \%$ \\
Durante o parto & 15 & $20,3 \%$ & 12 & $16,2 \%$ \\
No pré-parto & 23 & $31,1 \%$ & 4 & $5,4 \%$ \\
Gerenciarnento de Maternidade & 1 & $1,3 \%$ & - & - \\
\hline
\end{tabular}

Consideramos relevante separar as situações em que a entrevistada presta assistência rotineiramente daquelas em que essa assistência é realizada eventualmente. Verificamos que, das enfermeiras que atuam sempre, 33,8\% estão presentes na assistência puerperal. Uma hipótese deve-se ao fato de ser esta a fase do ciclo gravídico puerperal assistido tanto pelas profissionais que trabalham nas Maternidades como por aquelas que atuam nas Unidades Básicas de Saúde (UBS), abrangendo o puerpério imediato e tardio, respectivamente. A segunda fase em que as enfermeiras mais atuam foi o ciclo do pré-parto $(31,1 \%)$, cuja freqüência não corresponde ao do parto, isso porque, em duas das Maternidades incluídas, as enfermeiras não realizam o parto, mas sim, o controle da parturiente e o acompanhamento durante a execução do parto realizado pelo médico.

Podemos observar, também, que, entre as enfermeiras que atuam no puerpério, $56,7 \%$ referiram fazê-lo eventualmente. Esse fato pode ser explicado devido ao baixo índice de retornos das puérperas às UBS e à ausência de conexão entre a assistência pré e pósnatal. Essa atividade da Enfermagem precisaria ser resgatada, pois, certamente, poderia minorar a morbimortalidade infantil e promover o aleitamento materno mais prolongado.

Um dado que nos chamou a atenção foi o de que as enfermeiras atuam muito pouco e de forma descontínua na assistência pré-natal. Essa atividade apareceu muito mais como eventual. É rotina estabelecida para as Unidades Básicas de Saúde que a Enfermeira deve realizar a primeira consulta pré-natal, fazendo a matrícula da mulher no serviço e solicitando os exames laboratoriais preconizados. As consultas seguintes são agendadas, alternadamente, para o médico e para a enfermeira, mesmo tratando-se de uma gravidez de baixo risco. Somos da opinião de que a gestação de baixo risco poderia ser acompanhada pela Enfermeira (Obstétrica, se possível), encaminhando a gestante para a consulta médica, se houver intercorrências, mantendo-se as duas consultas com o médico obstetra, de acordo com as Normas e Manuais Técnicos para a Assistência PréNata ${ }^{(8)}$. É importante, também, que se garanta o preparo psico-físico da gestante para o parto, atribuição que a Enfermeira pode realizar com eficiência, o que aumenta a possibilidade de um parto eutócico.

Interessou-nos, também, verificar quais os 
principais problemas sentidos no seu cotidiano (Tabela 5). A falta de habilidade/segurança para a realização da consulta em pré-natal foi um dos mais referidos. Esse fato é decorrência do que foi comentado anteriormente, ou seja, da atuação descontínua da enfermeira nessa fase do ciclo gravídico-puerperal. É muito difícil termos segurança no desempenho de uma função que não praticamos com regularidade. Evidentemente, a segurança surge da prática que estimula a busca de conhecimento o qual, por sua vez, embasa essa prática.

Tabela 5 - Problemas apontados pelas enfermeiras que trabalham na assistência à mulher no ciclo gravídicopuerperal, Sorocaba-SP, 1999

\begin{tabular}{|c|c|c|}
\hline Problemas & II & $\%\left({ }^{*}\right)$ \\
\hline $\begin{array}{l}\text { Falta de habilidadelsegurança pi consulta em pré- } \\
\text { natal }\end{array}$ & 17 & 23,0 \\
\hline $\begin{array}{l}\text { Restriçẫo de pessoal e material } \\
\text { Acúmulo de funçốes }\end{array}$ & $\begin{array}{l}14 \\
14\end{array}$ & $\begin{array}{l}18,9 \\
18,9\end{array}$ \\
\hline Relacionamento com outros serviços & 8 & 10,8 \\
\hline Planta física da instituiçẫo & 6 & 8,1 \\
\hline Médico obstetra nẫo disponivel para referência & 5 & 6,7 \\
\hline Falta de reconhecimento profissional na prática & 4 & 5,4 \\
\hline Impedimento para realizar algumas atribuiçôes & 4 & 5,4 \\
\hline $\begin{array}{l}\text { Desvinculaçẫo na assistência pré-natalipartol } \\
\text { puerpério }\end{array}$ & 3 & 4,0 \\
\hline Falta de reciclagem e atualizaçẫo & 2 & 2,7 \\
\hline $\begin{array}{l}\text { Limites legais para assistência (prescriçẫo de } \\
\text { medicamentos) }\end{array}$ & 2 & 2,7 \\
\hline Atuaçẫo descontínua da Enfermeira no pré-natal & 2 & 2,7 \\
\hline Nẫo gosta da especialidade & 2 & 2,7 \\
\hline Falta de material e medicamentos atualizados & 1 & 1,3 \\
\hline Sabotagem e disputa de poder & 1 & 1,3 \\
\hline Problema de gênero (é homem) & 1 & 1,3 \\
\hline
\end{tabular}

(*) Considerou-se em $100 \%$ o número total de enfermeiras entrevistadas (74)

Uma das razões que também dificulta a atuação sistemática da enfermeira no pré-natal é o acúmulo de funções (18,9\% referiram este problema). Sabemos que, mesmo não estando na coordenação da UBS, a enfermeira tem sempre, sob seu encargo, vários programas, tais como o da Criança, da Imunização, do Hipertenso, de Diabéticos, entre outros. Seria desejável a criação do cargo de Enfermeira Obstétrica, permitindo que essa profissional pudesse se dedicar, exclusivamente, ao programa de Saúde da Mulher - ou Materno-Infantil. Lembramos a prioridade dessa área para a saúde de uma população.

Além do acúmulo de funções, outro problema citado foi a restrição de pessoal e material. Essas queixas são provenientes tanto das profissionais de Instituições hospitalares como das que atuam nas UBSs, corroborando o que já sabemos sobre a precariedade de recursos nos serviços de assistência à saúde.
A satisfação profissional foi uma outra variável que motivou este estudo. Verificamos que $73,0 \%$ das enfermeiras entrevistadas sentem-se satisfeitas no trabalho que realizam. Apontam, como motivos, a satisfação em trabalhar com a população, particularmente em programas que assistem a mulher, e, principalmente, o exercício de atividades assistenciais.

As que se sentem parcialmente satisfeitas, $23,0 \%$ do total, referiram como causas relevantes o desvio de atribuições (necessidade de desenvolver outras atividades que não as específicas e por terem que assumir, também, outras unidades além da sua, no caso da Enfermeira hospitalar), a falta de credibilidade e de reconhecimento do trabalho da enfermeira obstétrica, o pouco investimento (da Instituição) na área, a falta de autonomia profissional, o gasto excessivo de tempo com o gerenciamento da Unidade, o salário incompatível com a responsabilidade e, por fim, a pouca interação entre 0 grupo de trabalho.

Três enfermeiras referiram não se sentirem satisfeitas em trabalhar na área da Obstetrícia. Quando questionadas sobre o motivo, alegaram as condições de trabalho (restrição de pessoal e material, acúmulo de funções...), a falta de atualização, desmotivação e a predominância de ações voltadas à resolução de problemas criados por outros profissionais da equipe de saúde.

É digno de nota que $71,6 \%$ declararam não ter participado de nenhum evento na área, nos últimos dois anos. Considerando que a grande maioria refere satisfação na atividade que realiza, era de se esperar maior motivação para o desenvolvimento pessoal na especialidade.

No entanto, na questão de número 15 do formulário, que trata das perspectivas para o futuro profissional, as enfermeiras referiram o desejo de realizar cursos de atualização ou especialização. Quanto a isso, inquiridas sobre o curso oferecido pela PUC, em Sorocaba, disseram ter conhecimento dele, porém, para muitas das entrevistadas, o horário de trabalho não lhes permitiria o acompanhamento de um curso dessa natureza, e referiram, também, dificuldades de ordem familiar para o cumprimento dos estágios previstos. Essa constatação nos motivou a refletir sobre a possibilidade de organizar um curso específico para aprimoramento em assistência pré-natal, uma vez que a necessidade atual dessas enfermeiras se restringe a esse campo. Nesse caso, a carga horária poderia ser menor e com 
distribuição mais adequada às possibilidades das interessadas.

Considerando a relevância do trabalho da enfermeira, na assistência à gestante, os resultados desta pesquisa sensibilizaram-nos sobremaneira. Sabemos que o número de médicos obstetras na rede municipal, no momento, é insuficiente para a demanda de gestantes nas UBSs, e que as condições oferecidas e o salário não são favoráveis à admissão de novos profissionais médicos, pelo menos em curto prazo. Enquanto isso, a agenda do serviço pré-natal avoluma-se. Problemas ginecológicos também têm sua solução adiada, porém a gestante tem um prazo determinado pela natureza de sua necessidade. $O$ adiamento de uma consulta pré-natal pode ter conseqüências irreparáveis para a mãe e/ou para o bebê.

Por outro lado, de acordo com dados do Ministério da Saúde, é esperado que $80 \%$ das gestações evoluam normalmente, sem intercorrências, podendo ter o acompanhamento da enfermeira especializada, habilitada ou capacitada nessa área. Ficaria disponível, dessa forma, espaço suficiente na agenda médica para gestações de risco ou para intercorrências gestacionais.

Portanto, é mais que oportuno e necessário que seja oferecida às enfermeiras interessadas da rede municipal de saúde a possibilidade de atualizarem/ aprimorarem conhecimentos e habilidades para assumirem a assistência ao pré-natal de baixo risco nas Unidades Básicas de Saúde. Torna-se necessária, também, uma reestruturação das ações da enfermeira nas UBSs, pois um dos problemas citados com relevância foi o acúmulo de funções dessa profissional. É evidente que a Enfermeira que tem a seu cargo a coordenação da Unidade, não terá condições de assumir consultas.

Considerando a prioridade da área maternoinfantil, sugerimos a criação de cargos para enfermeiras especialistas, que, direcionando suas funções, poderiam prestar assistência com a qualidade à qual a população tem direito.

\section{CONCLUSÕES}

Com relação aos objetivos deste trabalho, verificamos que:

1. As instituições que assistem a mulher gestante, parturiente e puérpera em Sorocaba, na ocasião em que foi realizada a pesquisa, são: 6 maternidades, 26 UBS, 4
Unidades de Pronto Atendimento, 1 Policlínica de especialidades e o Departamento de Enfermagem do CCMB-PUCSP;

2. 93 enfermeiras atuam nessas instituições, realizando ações relacionadas à Enfermagem Obstétrica. Desse grupo, foram entrevistadas 74 enfermeiras das quais $25,7 \%$ são especializadas, $25,7 \%$, habilitadas, e $4 \%$ são habilitadas e especializadas na área de Obstetrícia. Das restantes, 33,8\% são habilitadas ou especializadas em outras áreas, e 10,8\% são apenas graduadas. A maioria da amostra é egressa da PUC-SP, tanto do curso de graduação $(74,3 \%)$ como dos cursos de habilitação e especialização $(79,5 \%)$, sendo que $51,3 \%$ delas estão graduadas num período que vai de 11 a 20 anos atrás. $49,3 \%$ dessas profissionais trabalham em mais de uma instituição e, destas, 52\% atuam na área de Obstetrícia também na segunda instituição em que trabalham. A faixa etária predominante entre as respondentes foi de 31 a 40 anos, e $62,2 \%$ delas são casadas, não atuando nenhum homem em Sorocaba;

3. Quanto à prática das enfermeiras entrevistadas, verificamos que, em relação ao ciclo gravídico-puerperal, $33,8 \%$ atuam mais freqüentemente na fase do puerpério, enquanto $56,7 \%$ o fazem apenas eventualmente, $48,6 \%$ atuam no pré-natal, porém eventualmente. Das entrevistadas, $31,1 \%$ referiram atuar sempre no pré-parto, porém apenas $20,3 \%$ assistem a mulher durante o parto, como atividade rotineira. Apenas uma enfermeira citou o gerenciamento da Maternidade como atividade exclusiva; 4. Quanto à satisfação profissional na atividade que executam, $73 \%$ manifestaram-se satisfeitas; 3 enfermeiras (4\%) referiram insatisfação com o trabalho atual, e os principais motivos apontados foram a falta de (condições para) atualização, a desmotivação e a predominância de atividades voltadas à resolução de problemas.

\section{RECOMENDAÇÕES}

A reflexão sobre a prática das enfermeiras, expressa nas entrevistas, levou-nos a três propostas concretas:

- Elaboração de um curso de aprimoramento/atualização com enfoque na assistência de enfermagem ao pré-natal de baixo risco, adequado às possibilidades das enfermeiras da rede básica municipal;

- Sugestão para a criação do cargo de Enfermeira 
Obstétrica na rede pública, possibilitando que a assistência pré-natal de baixo risco seja realizada rotineiramente por essa profissional;

- Participação do órgão formador (Universidade) na normatização das ações da Enfermeira Obstétrica nas instituições em que essa profissional atue.

\section{AGRADECIMENTOS}

Queremos expressar nossos agradecimentos às instituições envolvidas neste estudo, de maneira especial às colegas enfermeiras que atuam na assistência à mulher em Sorocaba, pela inestimável contribuição.

\section{REFERÊNCIAS BIBLIOGRAFICAS}

1. Silva GFM. A enfermagem obstétrica na realidade brasileira. Rev Bras Enfermagem 1965 outubro; 18 (4):270-86.

2. Rizzoto MLF. História da Enfermagem e sua relação com a Saúde Pública. Goiânia (GO): AB Editora; 1999.

3. Leis. Resolução n. 4 de 25 de fevereiro de 1972. Fixa os mínimos de conteúdo e duração do curso de graduação em enfermagem e obstetrícia. Documenta 1972; (140):566-8.

4. Riesco MLG. Perspectivas para a formação e qualificação profissional para assistência ao nascimento e parto no nível de graduação. II Seminário Estadual sobre o ensino de enfermagem para a assistência ao nascimento e parto, Sorocaba 19 e 20 de março 1999. Sorocaba (SP): Departamento Enfermagem.CCMB-PUCSP; 1999. p. 58-75. 5. Santos EF, Santos EB, Santana GO, Assis MF, Meneses RO Atos normativos do exercício de enfermagem. In: Legislação em Enfermagem: atos normativos do exercício e do ensino de enfermagem. São Paulo (SP): Atheneu; 1997. p 51-5.

6. Ministério da Saúde (BR). Portaria $n^{\circ}$ 2815/GM de 29 de maio de 1998. Trata da inclusão na tabela do Sistema de Informações Hospitalares do Sistema Único de Saúde (SIH/ SUS) de procedimentos e valores para assistência ao parto sem distócia por enfermeiro. Diário Oficial da União 1998 junho Seção 1, p.48.

7. Ministério da Saúde (BR). Portaria $n^{\circ} 163$ de setembro de 1998. Trata das atribuições do enfermeiro obstetra e da obstetriz. Diário Oficial da União 1998 setembro Seção 1, p. 24.

8. Ministério da Saúde (BR). Coordenadoria das Ações de Saúde da Mulher. Assistência Pré-Natal. Normas e Manuais Técnicos. 3. ed. Brasília (DF): Ministério da Saúde; 1999. 